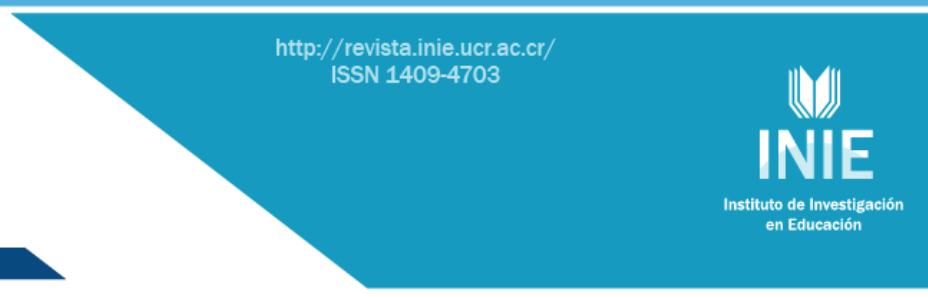

\title{
AUTOPERCEPCIÓN DE HABILIDADES DE LECTURA CRÍTICA EN ESTUDIANTES DE CIENCIAS DE LA EDUCACIÓN
}

SELF-PERCEPTION OF CRITICAL READING SKILLS OF STUDENTS IN EDUCATIONAL SCIENCES

Volumen 16, Número 1

Enero - Abril

pp. 1-23

Este número se publicó el $1^{\circ}$ de enero de 2016

DOI: http://dx.doi.org/10.15517/aie.v16i1.21713

\author{
Juan Pablo Díaz \\ Aníbal Roque Bar \\ Margarita Cristina Ortiz
}

Revista indizada en REDALYC, SCIELO

Revista distribuida en las bases de datos:

LATINDEX, DOAJ, E-REVIST@S, IRESIE, CLASE, DIALNET, SHERPA/ROMEO, QUALIS, MIAR

Revista registrada en los directorios:

ULRICH'S, REDIE, RINACE, OEI, MAESTROTECA, PREAL, CLACSO 


\title{
AUTOPERCEPCIÓN DE HABILIDADES DE LECTURA CRÍTICA EN ESTUDIANTES DE CIENCIAS DE LA EDUCACIÓN \\ SELF-PERCEPTION OF CRITICAL READING SKILLS OF STUDENTS IN EDUCATIONAL SCIENCES
}

\author{
Juan Pablo Díaz \\ Aníbal Roque Bar \\ Margarita Cristina Ortiz ${ }^{3}$
}

\begin{abstract}
Resumen: Este artículo propone identificar habilidades de lectura crítica autopercibidas por estudiantes de Ciencias de la Educación de una universidad pública de la ciudad de Resistencia (Chaco - Argentina). Se trabajó con una muestra de sesenta alumnos del cuarto y quinto nivel del Profesorado y la Licenciatura en Ciencias de la Educación, aplicando un cuestionario estructurado (CPC2), elaborado y estandarizado por Santiuste Bermejo. Si bien dicho instrumento indaga las percepciones respecto de las habilidades de lectura, escritura y verbalización, en esta ocasión se investigó sólo el primer tópico. Los ítems seleccionados para el análisis de la información se agruparon conforme con los procesos cognitivos que se describen en cada caso: en primera instancia, aquellos que dan cuenta de habilidades vinculadas con el análisis previo a lectura; y en la segunda, en relación con el propio examen crítico. Los resultados muestran que la mayoría de los estudiantes señala reconocer ideas relevantes y tesis, realizar inferencias, valorar soluciones, apreciar la vigencia del contenido y los argumentos para la toma de decisiones fundadas. No obstante, se observa cierta ambigüedad en relación con la habilidad para acordar o no con la postura de los autores. Estos hallazgos son consistentes con lo esperado de estudiantes de carreras multiparadigmáticas.
\end{abstract}

Palabras clave: HABILIDADES DE LECTURA CRÍTICA, AUTO-PERCEPCIÓN, ESTUDIANTES AVANZADOS, UNIVERSIDAD, ARGENTINA.

\begin{abstract}
This article intends to identify self-perceived critical reading skills in advanced students of Educational Sciences in a public university in Resistencia (Chaco-Argentina). Sixty students from the fourth and fifth levels of the courses of studies for teachers and a Licentiate Program in Educational Sciences, were recruited for the study and requested to answer a structured questionnaire (CPC2), which was designed and standardized by Santiuste Bermejo. Although this instrument investigates the perceptions related to reading, writing and oral skills, at this stage we investigated only the first topic. In order to analyze the information, the items selected were grouped according to the cognitive processes required for critical reading: firstly, those related to the skills before reading; and secondly, those related to the critical assessment itself. The results of the research shows that most students recognize relevant ideas and thesis, make inferences, ponder over solutions, and appreciate the dateness of the content and the arguments to make informed decisions. However, a certain ambiguity can be observed in relation to their skill to agree or disagree on the authors' attitude towards the subject. These findings conform to what is expected of students from multi-paradigmatic courses of studies.
\end{abstract}

Keywords: CRITICAL READING SKILLS, SELF-PERCEPTION, ADVANCED STUDENTS, UNIVERSITY, ARGENTINE.

1 Docente universitario. Profesor en Ciencias de la Educación. Universidad Nacional del Nordeste, Argentina. Auxiliar Docente de Primera Categoría. Becario de Investigación.Dirección electrónica: pablodiaz@hum.unne.edu.ar

2 Docente Investigador. Doctor en Ciencias Cognitivas. Universidad Nacional del Nordeste, Argentina. Profesor Titular Regular. Dirección electrónica: anibalrbar@hum.unne.edu.ar

3 Docente Investigadora. Universidad Nacional del Nordeste, Argentina. Profesora Adjunta Regular. Magister en Epistemología y Metodología de la Investigación. Dirección electrónica: mortizgaleano11@gmail.com

Artículo recibido: 10 de marzo, 2015

Enviado a corrección: 26 de mayo, 2015

Aprobado: 26 de octubre, 2015

Volumen 16 Número 1, Año 2016, ISSN 1409-4703 


\section{Introducción}

El ámbito universitario es el lugar propicio para el desarrollo de profesionales competentes, independientemente del campo disciplinar y profesional. United Nations Educational, Scientific and Cultural Organization (UNESCO, 1999) y otros organismos internacionales plantean la necesidad de fortalecer y hacer hincapié en el desarrollo de pensamiento crítico en todos los niveles, particularmente en el superior, para formar ciudadanos reflexivos y capaces de enfrentar situaciones al evaluar sus posibles soluciones.

Esto es fundamental en carreras multiparadigmáticas (Biglan, 1973) como Ciencias de la Educación, en las que el abordaje de un problema requiere apelar a distintas alternativas teóricas por la complejidad de lo educativo como hecho social.

El fomento y desarrollo del pensamiento crítico radica además en una razón sustancial: la formación de futuros educadores, que a su vez, actuarán como formadores de otros profesionales. Asimismo, la lectura crítica es la herramienta para el logro de un pensamiento de orden superior y de sujetos comprometidos con la transformación de su contexto social.

En el trayecto de la formación universitaria en los distintos campos disciplinares los estudiantes incorporan múltiples habilidades cognitivas, entre ellas destacan especialmente aquellas vinculadas a la escritura, la lectura y al hablar y escuchar (Santiuste-Bermejo, 2001).

Los debates actuales sobre la formación universitaria y, particularmente sobre la enseñanza y la utilización del pensamiento crítico plantean esta cuestión como clave para ser pensada e investigada en distintos ámbitos (Lipman, 1998; Paul y Elder, 2005; Hawes, 2003; UNESCO, 1999).

Pese a la importancia atribuida al estudio de esta problemática en el marco de la literatura especializada, el desarrollo de habilidades de lectura crítica en estudiantes avanzados en el nivel superior resulta un área de investigación poco explorada. No obstante, se hallaron indagaciones realizadas con alumnos universitarios (Marciales-Vivas, 2003; Maglio y Luque, 2011; Curone, Alcover, Pabago, Martinez-Frontera, Mayol y Colombo, 2011). Sobre la base de las contribuciones de estas investigaciones dedicadas al análisis del pensamiento crítico, las competencias y habilidades de lectura crítica y autopercepción, se pretende aportar desde la especificidad disciplinar de un profesorado en Ciencias de la Educación, este lugar de vacancia. 
Durante su formación académica, los estudiantes avanzados habrían de familiarizarse con distintas herramientas teóricas y metodológicas propias de sus disciplinas, lo que posibilitaría su futura inserción profesional.

Este trabajo plantea como hipótesis la autopercepción positiva de los estudiantes en tanto lectores críticos, es decir, cabría esperar una valoración satisfactoria del grado de desarrollo de este tipo de habilidades cognoscitivas. Específicamente, el objetivo es identificar las habilidades de lectura crítica auto-percibidas por estudiantes avanzados de Ciencias de la Educación de una universidad pública. La investigación se desarrolló en el marco de un proyecto de investigación mayor que indagó las "Habilidades de razonamiento y creencias epistemológicas de estudiantes avanzados en contextos académico-disciplinares" (PICTO 2007, UNNE 00175).

Se parte de la premisa de la existencia de una diversidad de trabajos acerca de la lectura crítica desde distintos enfoques. La perspectiva psicolingüística, refiere a los procesos cognitivos que permiten que la información visual se convierta en información verbal y luego sea transferida hacia los niveles superiores donde adquiere significado (Marciales-Vivas, 2003; Yudkin, 2006; Serrano y Madrid, 2007; Serrano de Moreno, 2008); mientras que las indagaciones de tipo sociocultural, sostienen la lectura y la escritura como construcciones sociales, actividades socialmente definidas (Luke y Freebody, 1997; Cassany, 2006). Nuestra propuesta aborda las mencionadas habilidades desde una perspectiva psicocognitiva, en la que la comprensión lectora requiere del desarrollo de destrezas mentales o procesos cognitivos. Es decir, pensar la lectura como un proceso activo, constructivo e interactivo, donde el lector emplea conocimientos previos para interactuar con el texto y así, construir significados o sentidos.

\section{Breve referencia teórica}

UNESCO (1999) plantea profundos cambios en la sociedad actual y encuentra en la educación un canal de respuestas bajo el supuesto de que esta es motor de transformación. Incluye todos los niveles del sistema educativo, pero focaliza la formación universitaria por su liderazgo innovador en la formación de los profesionales comprometidos y participativos que requiere el siglo $\mathrm{XXI}$, acorde con sus metas.

A lo largo de la formación escolar, los sujetos deberían adquirir determinadas habilidades a fin de alcanzar otras de nivel superior más complejas. En este sentido, la formación universitaria apuntaría a la promoción de las diferentes capacidades propias del 
pensamiento crítico; esto es, no sólo en relación con las competencias para el futuro ejercicio profesional, sino también en función de la necesaria formación de ciudadanos críticos, reflexivos y democráticos.

La Universidad es per se la institución promotora de estrategias de pensamiento crítico, tanto en las dimensiones de lectura como de escritura y comunicación oral, conforme sean las carreras monoparadigmáticas o multiparadigmáticas (Biglan, 1973). En la última, se encuadran las Ciencias de la Educación, campo disciplinar en el que se realizó el estudio sobre el pensamiento crítico y particularmente, las habilidades de lectura crítica autopercibidas por los estudiantes.

Esta perspectiva hace referencia a la capacidad humana de comprender la realidad como tal, distinguiéndola de lo que no es.

El pensamiento crítico es entendido como "aquella forma de pensamiento acerca de cualquier tema, contenido o problema en el cual el individuo mejora la calidad de sus ideas al apoderarse cuidadosamente de las estructuras inherentes al pensamiento e imponiendo sobre ellas patrones intelectuales" (Paul y Elder, 2003, p. 75). Es auto-correctivo (relacionado con la metacognición), sensible al contexto, orientado por criterios y lleva a juicios (Lipman, 1998, p.174).

En esta línea, Paul y Elder (2005) aluden al proceso metacognitivo por el cual cada sujeto evalúa y enriquece el desarrollo de sus capacidades de pensamiento hacia el desenvolvimiento de la creatividad y criticidad (autorregulación).

Por su parte, Herrera-Ucrós y Villalba-Mercado (2012) lo diferencian del pensamiento cotidiano expresando que: "(...) Plantearse interrogantes sobre el asunto de interés, recopilar información, analizar conceptos, premisas, puntos de vista, comprender las implicaciones, consecuencias y llegar a formular conclusiones y soluciones, son características evidentes de la persona que hace buen uso del pensamiento crítico" (p. 55 ).

Betancourth-Zambrano, Insuasti-Quevedo y Riascos-Portilla (2012) plantean y definen las habilidades de pensamiento crítico (Proyecto Delphi, 1990) y las indagan en estudiantes universitarios de psicología. Concluyen que la inferencia, la explicación y los puntos de vista fueron los reconocidos y señalados como altamente significativos y que este tipo de pensamiento se aplica a todo lo susceptible de ser pensado, analizado, valorado y evaluado desde la lógica de la razón.

Para Ennis (2002), un pensador crítico es aquel que:

a. Propone explicaciones, hipótesis, planes u otras fuentes alternativas. 
b. Asume una posición frente al texto leído y busca otros fundamentos (políticos, ideológicos, religiosos, entre otros) cuando este no brinda suficientes evidencias o puntos de vista.

c. Dispone de información actual sobre el propio campo disciplinar.

d. Está abierto a otros puntos de vista.

Asimismo, la autora destaca el valor de las capacidades de identificar, explicar, reconocer y distinguir alternativas como medios de fortalecer la alfabetización académica para la activa participación en el discurso de la disciplina y en el análisis, interpretación y producción de textos en el nivel universitario. Es decir, habilidades para evaluar información, visualizar relaciones entre las ideas y utilizarlas como apoyo en la lectura crítica.

Se concibe la lectura crítica como un proceso de hacer juicios, evaluar la relevancia y exactitud de lo que se lee, un acto en el cual se usa una actitud de cuestionamiento, análisis lógico e inferencia para juzgar lo leído de acuerdo con un estándar establecido. (Campos Arena, 2007 citado en Herrera-Ucrós y Villalba-Mercado, 2012).

De acuerdo con Furedy y Furedy (1985), se transfieren a la lectura crítica las siguientes habilidades cognitivas del pensamiento crítico: identificar vínculos relevantes, analizar consecuencias, realizar inferencias correctas, valorar pruebas y propuestas consistentes.

Estas habilidades han sido estudiadas por Marciales-Vivas (2003), focalizando las de lectura crítica en los cursantes de carreras de índole multiparadigmática (Filosofía y Psicología, de una universidad colombiana), mediante la aplicación del Cuestionario de Pensamiento Crítico (CPC2) adoptado en este trabajo. Los resultados denotan el alto grado de este tipo de lectura en los estudiantes, reflejado en el análisis de las fuentes, la evaluación de ventajas e inconvenientes de las soluciones a un problema, la justificación de las conclusiones, la diferenciación entre hechos y opiniones y el reconocimiento de evidencias a favor y en contra de una tesis propuesta por el autor en textos argumentativos.

Por su parte, Curone; Alcover; Pabago; Martinez-Frontera; Mayol y Colombo (2011) indagaron el pensamiento crítico con el mismo instrumento en ingresantes a la carrera de Psicología de la UBA, dando cuenta que la mayoría no tuvo dificultades para distinguir lo principal, hechos de opiniones, extraer conclusiones y evaluar la vigencia del texto. No obstante, estas emergieron en relación con el reconocimiento de la lógica interna del texto, la 
ponderación de soluciones, sus condiciones y viabilidad práctica y el cuestionamiento de las interpretaciones propuestas.

Estas capacidades o competencias de pensamiento crítico tienen en el ámbito universitario un lugar predominante y son objeto de estudio (Alsina y Almirón, 2013; CandelGutiérrez y Pareja-Candel, 2013; Gómez-Puerta, Roca-Uberes y Guerrero-Solé, 2014).Es justamente, en los procesos de construcción del conocimiento, cuando el lector desarrolla dichas competencias al ponerlas en acción, generalmente sin percibirlas o tener conciencia de ello. La percepción de estas reseña creencias relativas de los estudiantes respecto de su capacidad para movilizar y organizar recursos propios en la realización de acciones específicas a fin de alcanzar determinados resultados (Cunha, Mesquita, Moreno, Boleto, Tavares y Silva, 2010 citado en Delgado-Martínez y Becerra-Álvarez, 2012).

En este estudio, la competencia indica tanto el buen desempeño alcanzado como la capacidad para actuar exitosamente en el futuro (Masten y Coatsworth, 1998). Concretamente la lectura crítica es potenciadora en la construcción de sentido, lo que colabora significativamente en la elaboración de representaciones sobre el contenido disciplinar. Dichas representaciones incluye lo un lector critico deber saber cómo lo tiene que hacer y por qué lo debe hacer (Serrano de Moreno y Madrid, 2007), e involucran conocimientos lingüísticos y culturales, habilidades, actitudes y valores del contexto social (Hymes, 1996; Vigotsky, 1985), como también, estrategias cognitivas y metacognitivas.

Las últimas han sido inquiridas por Herrera-Ucrós y Villalba-Mercado (2012) en estudiantes universitarios de la Facultad de Ingeniería (Universidad de Sucre, Colombia). Entre los rasgos de lectura crítica encontrados, señalan los referidos a la evaluación de fuentes; el reconocimiento de las intenciones del autor, de falacias, contradicciones, estereotipos; la diferenciación entre hechos y opiniones, entre otros. Indican también haber observado dificultades en la interpretación global del texto; identificación de hipótesis y de relaciones de comparación y contraste; evaluación de la confiabilidad (hechos, opiniones e inferencias); reconocimiento del tipo de lenguaje utilizado, objetivos del autor y de elementos tendenciosos del texto, lo que a su juicio dificulta la construcción de valoraciones y posiciones críticas.

En este sentido, se asume con Serrano de Moreno (2008) que el estudiante como lector crítico no acepta a priori las ideas; identifica diferentes puntos de vista, intenciones e implicaciones; analiza el sentido profundo del texto, las ideas subyacentes, razonamientos e ideología; reconoce el contenido sustantivo y cuestionador de las fuentes a las que accede. 
Las citadas habilidades de lectura crítica constituyen el foco del trabajo, aunque desde la auto-percepción de los estudiantes. Es decir, las valoraciones que tienen respecto de sus capacidades relacionadas con sus creencias, actitudes, deseos, expectativas que se fundamentan en la realidad y se transforman en su mundo interior (Martínez, 2009).

Galindo-Ruiz de Chávez (2015) indagó las características de las habilidades de lectura crítica hipertextual en la WEB 2.0 auto-percibidas en una muestra aleatoria de estudiantes avanzados (Ciencias Sociales y Humanas, entre otros) de la Universidad de Sonora. La mayoría de los estudiantes asume que las tareas críticas más desarrolladas por ellos son, las estrategias de orden superior como análisis, síntesis y evaluación. Con menor recurrencia aluden a la recopilación, comprensión y aplicación de información; las menos seleccionadas fueron determinar propósitos y realizar inferencias.

Un aporte significativo es el estudio de Maglio y Luque (2011) quienes analizaron la percepción que de sí mismos tienen los estudiantes de $3^{\circ}$ año de psicología (Profesorado y Licenciatura) de la Universidad Nacional de La Plata, como lectores y escritores. Más de la mitad manifestó poseer dificultades en la lectura vinculadas con el reconocimiento del vocabulario técnico e ideas principales, la comprensión y la realización de inferencias.

Para Mota de Cabrera (2010), este tipo de lectores debe reconocer las ideas relevantes, centrarse en el tema o problema y hacer preguntas o reformularlas con el fin de encontrar información pertinente que responda a la situación problema; tal como postulan Argudín y Luna (1994):

(...) sabe seleccionar la información importante de la irrelevante. La habilidad implica: reconocer los asuntos centrales o ideas clave (puntos principales) además de saber identificar los enunciados de apoyo; distinguir cómo está organizado un texto e identificar las contradicciones internas del texto, así como las argumentaciones ambigüas o tendenciosas. (p. 27)

Además, todo buen lector es capaz de "mirar" entre líneas, captar las intencionalidades y entender la lógica interna del texto (Hawes, 2003).

De acuerdo con lo expuesto, el abordaje del pensamiento crítico y lectura crítica se realiza desde una perspectiva psicocognitiva, con el objetivo de identificar las habilidades de lectura crítica auto-percibidas. 


\section{Metodología}

El diseño metodológico de la investigación es exploratorio-descriptivo. La muestra $(n=60)$ estuvo integrada por estudiantes avanzados del cuarto y quinto nivel de la carrera del Profesorado y de la Licenciatura en Ciencias de la Educación de una universidad de gestión pública (República Argentina).

En la tabla 1 se incluye las características de la muestra de estudiantes participantes en la investigación:

Tabla 1: Descripción de la población

\begin{tabular}{|c|c|c|c|c|c|}
\hline \multirow[t]{2}{*}{ Variables } & \multicolumn{2}{|c|}{$\%$ de Sexo } & \multirow{2}{*}{$\begin{array}{l}\text { Rango } \\
\text { Etario }\end{array}$} & \multirow[t]{2}{*}{$4^{\circ}$ nivel } & \multirow[t]{2}{*}{$5^{\circ}$ nivel } \\
\hline & Femenino & Masculino & & & \\
\hline Profesorado & 24 & 11 & $22-24$ & 23 & 12 \\
\hline Licenciatura & 19 & 6 & $25-27$ & 16 & 9 \\
\hline
\end{tabular}

Fuente: Elaboración propia

Para la recolección de información se aplicó un cuestionario de pensamiento crítico (CPC2) elaborado por Santiuste-Bermejo, Barrigüete, García, González, Rossignoli y Toledo (2001). Este instrumento estandarizado releva la autopercepción sobre este tipo de pensamiento cuando se ejecutan tareas de lectura, escritura, escucha y habla. Consta de treinta afirmaciones con cinco opciones de respuesta (Total desacuerdo, Desacuerdo, A veces, Acuerdo, Total acuerdo), que el estudiante selecciona previa valoración de éstas. Cabe señalar, por un lado, que para este trabajo solamente se analizaron dieciséis (16) ítemes referidos a las habilidades de lectura crítica y por otro, que las siglas "D.A."; "A.A." y A.V, incluyen las respuestas Total desacuerdo y desacuerdo; Total acuerdo y acuerdo y $A$ veces, respectivamente.

Los ítemes seleccionados se agruparon y analizaron en dos bloques, conforme con los procesos cognitivos que demanda la lectura crítica de cualquier texto; el primer bloque agrupa acciones que buscan identificar y diferenciar las partes del escrito para su posterior tratamiento (Análisis previo al trabajo crítico). En tanto que el segundo involucra tarea que refieren al examen crítico propiamente dicho.

\section{Resultados}

Se presentan las diferentes habilidades que los estudiantes avanzados de Ciencias de la Educación declaran al realizar los procesos de análisis previo y de examen crítico del contenido cuando leen. Estos implican estrategias cognitivas y metacognitivas, pero dado 
que escapan a los alcances del estudio se focaliza la interpretación de los datos del Cuestionario de Pensamiento Crítico (CPC2) en torno a las Habilidades de Lectura Crítica, a fin de obtener una aproximación a dichas capacidades.

El análisis previo involucra habilidades que pueden caracterizarse como básicas y necesarias para luego examinar críticamente el contenido, proceso que incluye otras más complejas y que, en conjunto, posibilitarían el tránsito hacia el pensamiento crítico.

\section{Análisis previo en el trabajo crítico}

La tabla 2 muestra la cantidad de ítemes relacionados con las tareas previas a la lectura en sí misma y el porcentual promedio de los valores obtenidos en cada habilidad.

Tabla 2: Bloque I: Análisis previo al trabajo crítico

\begin{tabular}{|c|c|c|c|c|c|}
\hline Habilidades & \multicolumn{3}{|c|}{ Identificación de las partes } & \multicolumn{2}{|c|}{ Diferenciación de las partes } \\
\hline İtemes & 30 & 13 & 16 & 24 & 28 \\
\hline D. A. & 6,7 & 10 & 5 & 3,4 & 3,4 \\
\hline$A$ V V. & 15 & 26,7 & 30 & 15 & 28,3 \\
\hline A. $A$ & 78,3 & 63,3 & 65 & 81,6 & 68,3 \\
\hline $\begin{array}{c}\text { Promedio } \\
\%\end{array}$ & \multicolumn{3}{|c|}{$\begin{array}{l}\text { D. A: } 7,2 \\
\text { A.V: } 23,9 \\
\text { A. A: } 68,9\end{array}$} & \multicolumn{2}{|c|}{$\begin{array}{l}\text { D.A: } 3,4 \\
\text { A.V: } 21,6 \\
\text { A. A: } 75\end{array}$} \\
\hline
\end{tabular}

Fuente: Elaboración propia

Los gráficos 1, 2 y 3 ilustran las diferentes sub-habilidades que conforman la habilidad Identificación de las partes de un texto.

Gráfico 1: Identificación de información irrelevante

Ítem 13: Cuando leo un texto, identifico claramente la información irrelevante y prescindo de ella

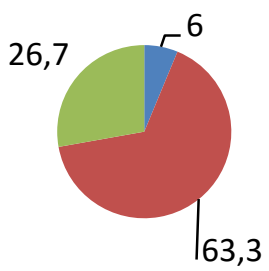

Desacuerdo con la afirmación

A veces

63,3

Fuente: Elaboración propia 
Dos tercios de la muestra indicaron identificar y no tener en cuenta la información carente de importancia al realizar la lectura de un texto de modo ocasional.

Gráfico 2: Identificación de argumentos de un texto

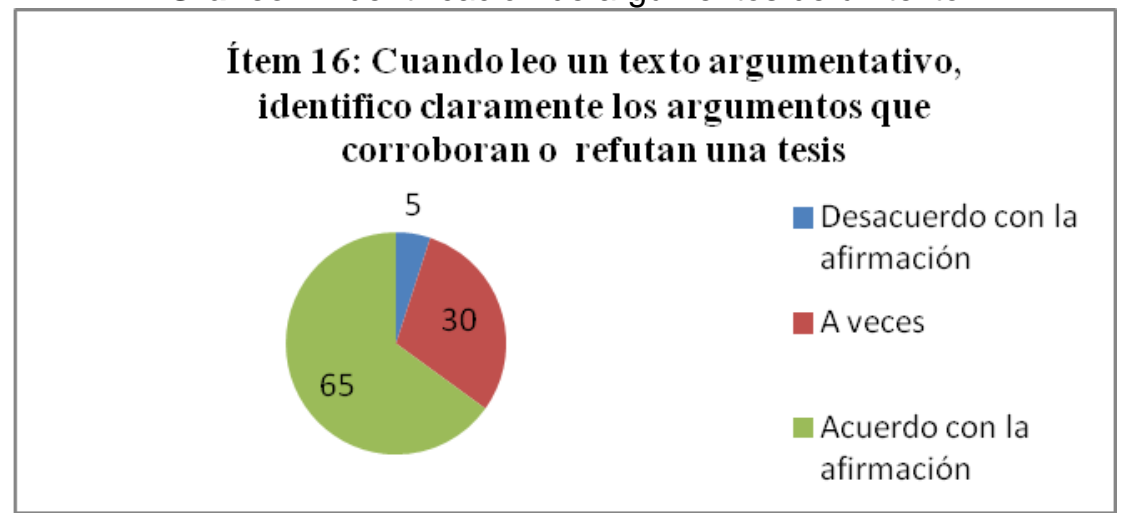

Fuente: Elaboración propia

Un significativo porcentual de estudiantes señaló reconocer con claridad los argumentos o razones que apoyan o invalidan la tesis en textos argumentativos.

Gráfico 3: Identificación de información relevante

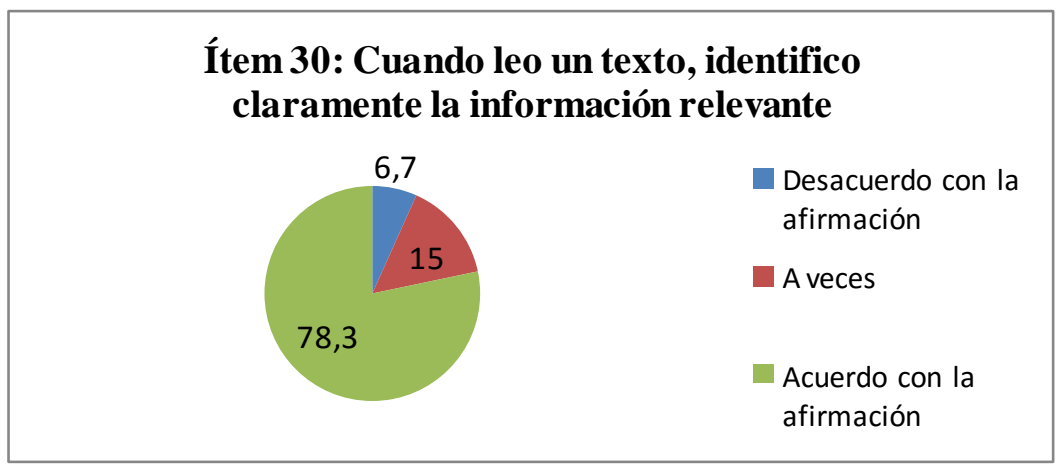

Fuente: Elaboración propia

La mayoría expresó que distingue fehacientemente la información significativa cuando lee un texto.

Los gráficos 4 y 5 ilustran las diferentes sub-habilidades que conforman la habilidad: Diferenciación de las partes. 
Gráfico 4. Diferenciación de opiniones

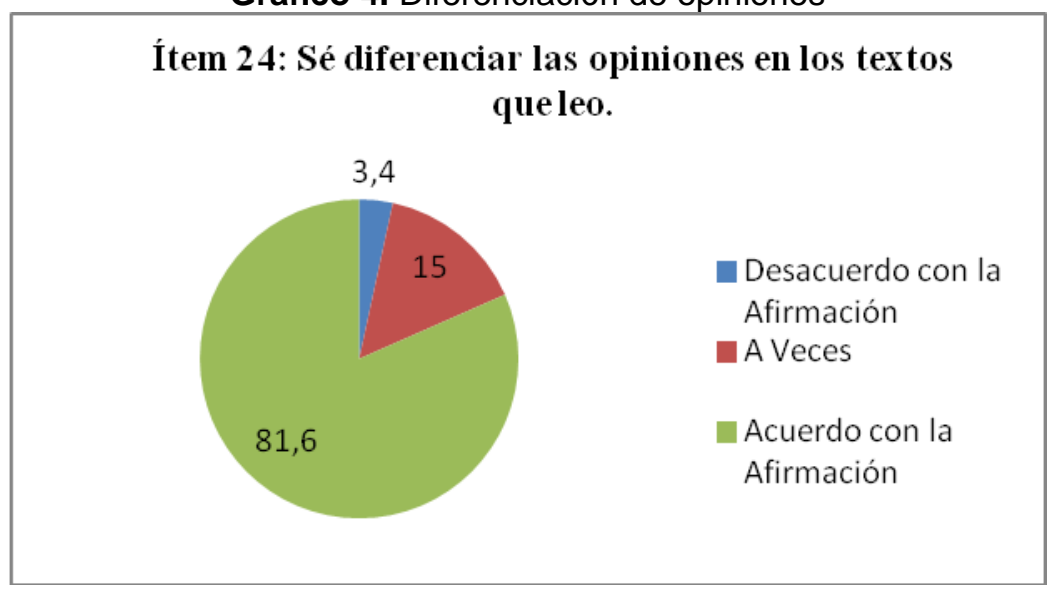

Fuente: Elaboración propia

Prácticamente la totalidad de los encuestados afirmó diferenciar las opiniones de los autores al leer textos.

Gráfico 5. Reconocimiento de opiniones, problemas, soluciones y explicaciones

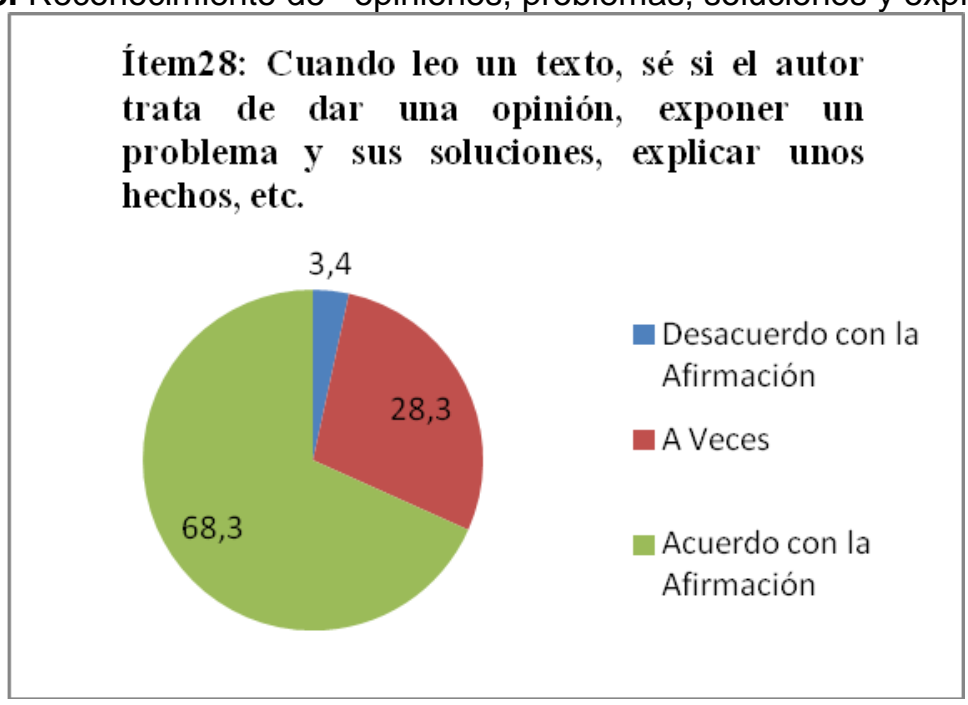

Fuente: Elaboración propia

La mayoría manifestó saber distinguir en un texto los enunciados referidos a opiniones, los que presentan un problema y soluciones y las proposiciones explicativas. 


\section{Examen crítico del contenido}

En la tabla 3 se muestran las selecciones de los estudiantes en relación con las habilidades críticas propiamente dichas.

Tabla № 2: Examen crítico propiamente dicho

\begin{tabular}{|c|c|c|c|c|c|c|c|c|c|c|c|}
\hline Habilidades & \multicolumn{2}{|c|}{$\begin{array}{c}\text { Elaboración } \\
\text { de } \\
\text { Inferencias }\end{array}$} & \multicolumn{6}{|c|}{ Valoraciones efectuadas sobre el texto } & \multicolumn{3}{|c|}{$\begin{array}{l}\text { Análisis de componentes para } \\
\text { acordar o desacordar }\end{array}$} \\
\hline & & & & & & & & & $\begin{array}{c}\text { Focalizado en } \\
\text { la producción } \\
\text { ajena. }\end{array}$ & $\begin{array}{r}\text { cen } \\
\text { con } \\
\text { entre } \\
\quad\end{array}$ & $\begin{array}{l}\text { is } \\
\text { en la } \\
\text { ación } \\
\text { copio y } \\
\text { ho. }\end{array}$ \\
\hline Ítemes & 21 & 7 & 1 & 19 & 11 & 18 & 25 & 12 & 2 & 22 & 17 \\
\hline D.A. \% & 3,3 & 3,3 & 1,7 & 8,4 & 10 & 11,7 & 8,3 & 6,7 & 41,7 & 5 & 13,3 \\
\hline A V. \% & 26,7 & 20 & 38,3 & 28,3 & 30 & 38,4 & 21,7 & 28,3 & 45 & 45 & 43,3 \\
\hline A. A.\% & 70 & 76,7 & 60 & 63,3 & 60 & 50 & 70 & 65 & 13,3 & 50 & 43,3 \\
\hline $\begin{array}{l}\text { Promedio } \\
\%\end{array}$ & \multicolumn{2}{|c|}{$\begin{array}{l}\text { D.A:3,4 } \\
\text { A.V: } 23,3 \\
\text { A.A: } 73,3\end{array}$} & \multicolumn{6}{|c|}{$\begin{array}{l}\text { D. A: } 7,7 \\
\text { A.V: } 30,8 \\
\text { A.A: } 61,4\end{array}$} & $\begin{array}{l}\text { D. A: } 41,7 \\
\text { A.V: } 45 \\
\text { A. A: } 3,3\end{array}$ & \multicolumn{2}{|c|}{$\begin{array}{l}\text { D.A:9,2 } \\
\text { A.V: } 44,2 \\
\text { A.A: } 46,6\end{array}$} \\
\hline
\end{tabular}

Fuente: Elaboración propia

Se discriminan las sub-habilidades constitutivas de la habilidad: Elaboración de inferencias, en los gráficos 6 y 7 .

Gráfico 6. Extracción de conclusiones

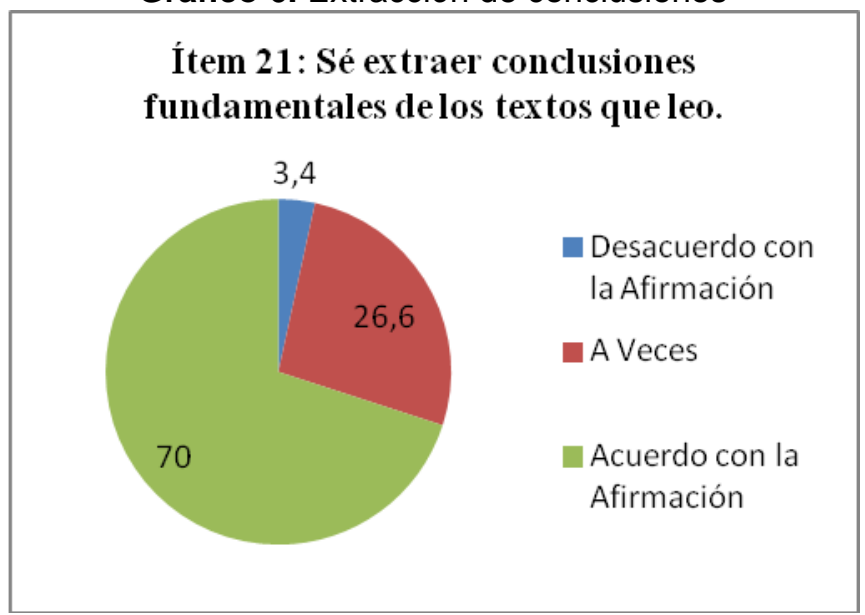

Fuente: Elaboración propia

El proceso de extraer las principales conclusiones de los textos objeto de lectura, es una capacidad percibida por un significativo porcentaje de los encuestados. 


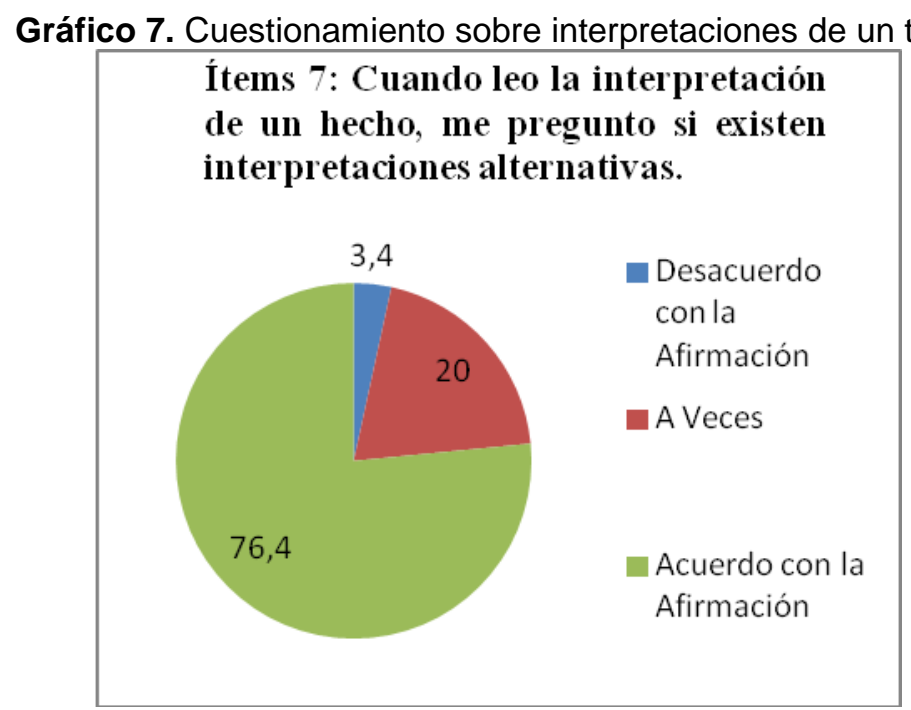

Fuente: Elaboración propia

Los estudiantes expresaron no ceñirse a la interpretación de hechos dada por el autor y buscar otras alternativas.

Los gráficos $8,9,10,11,12$ y 13 refieren las sub-habilidades de la habilidad: Valoraciones efectuadas sobre el texto.

Gráfico 8: Valoración de la utilidad de soluciones

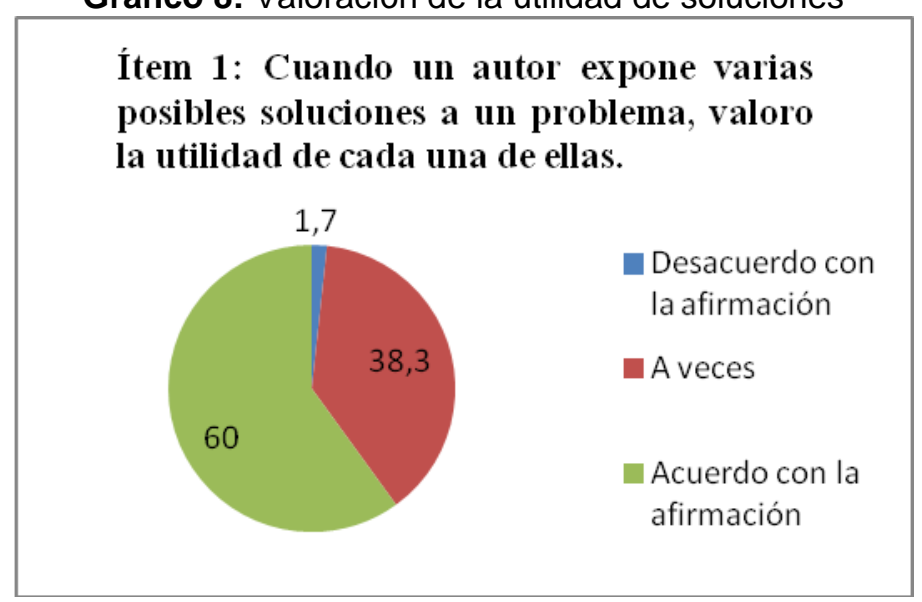

Fuente: Elaboración propia

Más de la mitad de la muestra indicó juzgar la utilidad de las soluciones a problemas propuestas por el autor, aunque una proporción próxima a la media, dijo hacerlo a veces. 
Gráfico 9: Valoración de las condiciones de soluciones

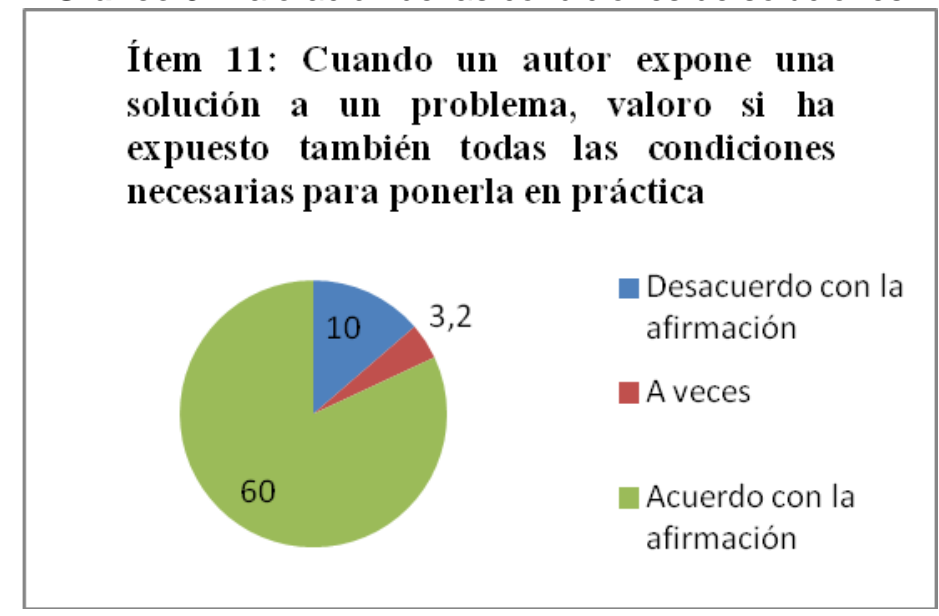

Fuente: Elaboración propia

También valuaron en idéntico porcentual al caso anterior, la explicitación de las condiciones requeridas para la puesta en práctica de la solución expuesta.

Gráfico 10: Valoración de la viabilidad de soluciones

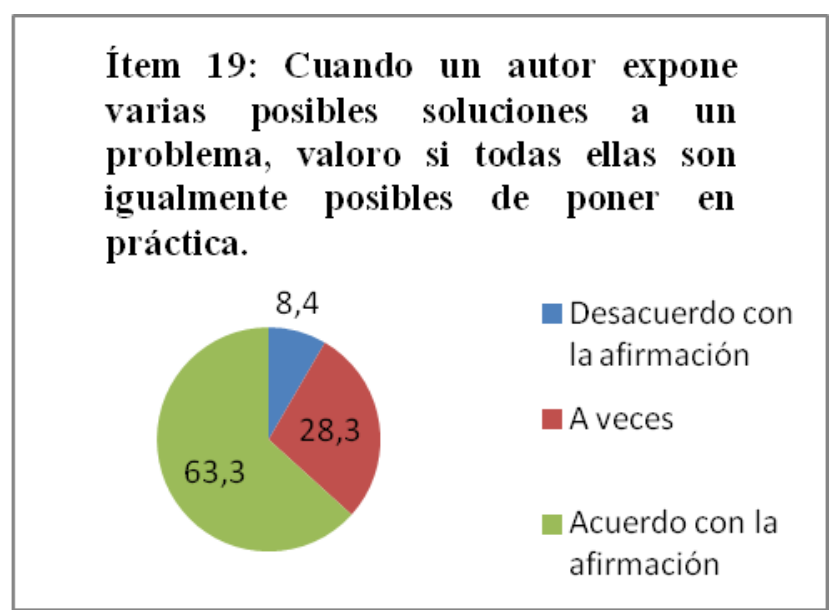

Fuente: Elaboración propia

Si bien la mayoría de los estudiantes se inclina por afirmar que valora posible soluciones a un mismo problema, la alta proporción de "a veces" en relación con los demás ítemes muestra mayor nivel de incertidumbre por parte de los sujetos que en otros casos. Esto podría estar indicando que las opiniones se sustentan más en lo debería hacerse que en situaciones reales donde estas decisiones se sustancian efectivamente. 
Gráfico 11: Examinar la articulación de ideas

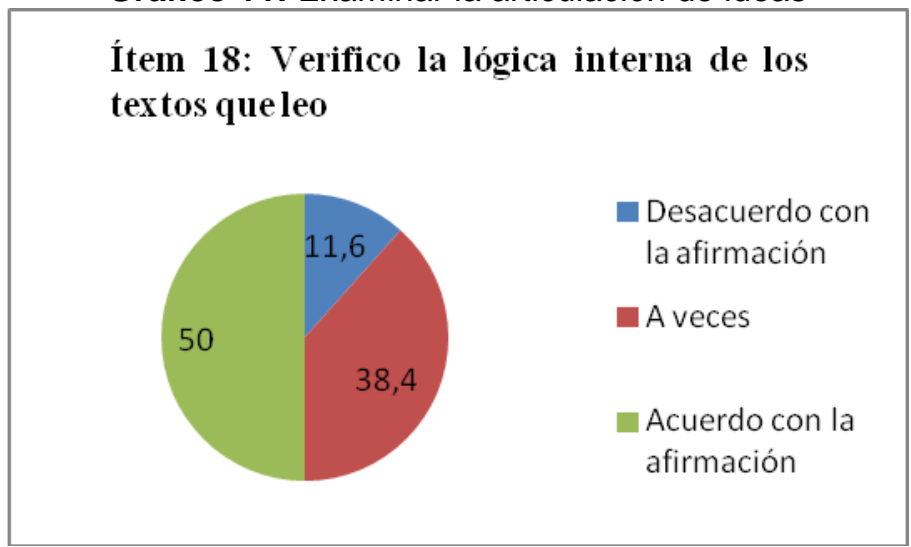

Fuente: Elaboración propia

Solo la mitad de los encuestados indicó estimar cuándo las ideas involucradas en un texto se articulan, apoyan entre sí y tienen sentido. Sin embargo, un porcentual cercano a este valor, únicamente a veces consideró dicha cuestión.

Gráfico 12: Evaluación de evidencias

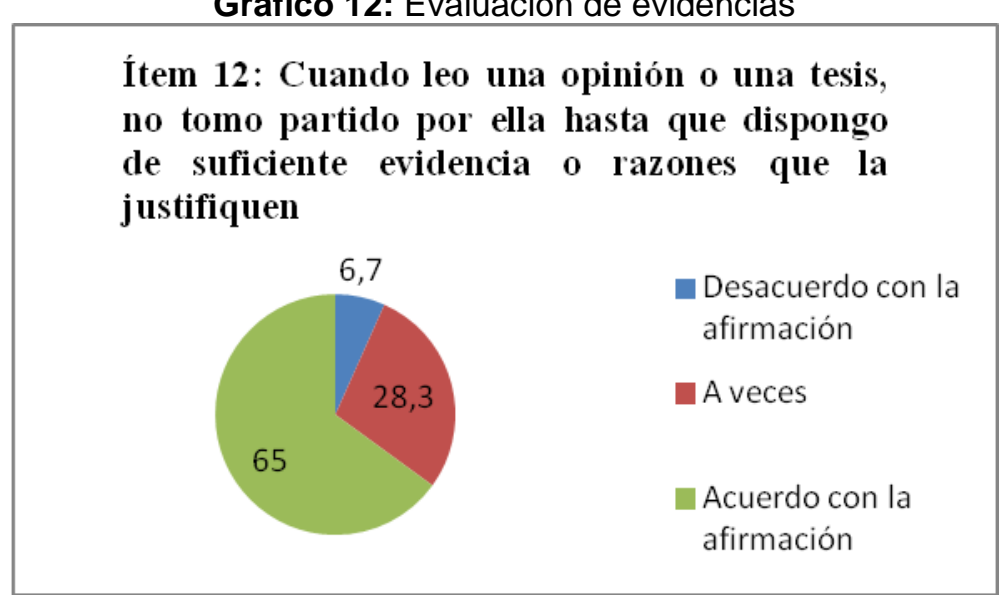

Fuente: Elaboración propia

Casi dos tercios de la muestra marcaron la necesidad de contar con argumentos que apoyen una tesis u opinión antes de adoptarla, percepción sostenida ocasionalmente por un tercio de los estudiantes. 
Gráfico 13: Valoración de la fuente

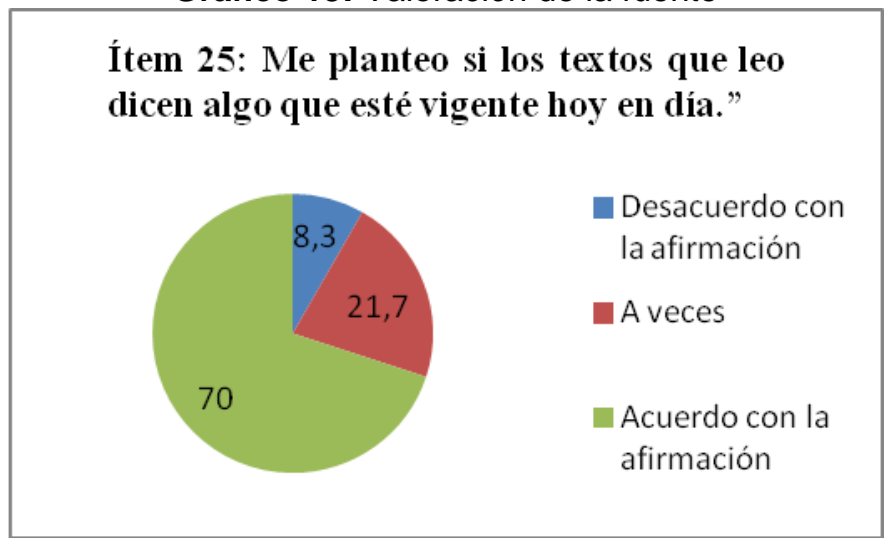

Fuente: Elaboración propia

Una significativa cantidad de estudiantes refirió juzgar el grado de actualización de la información provista por los textos a los que acceden.

En los gráficos 14, 15 y 16 se expone la habilidad Análisis de componentes para acordar o desacordar, contemplando las sub-habilidades: a) Análisis focalizado en la producción ajena y b) Análisis centrado en la confrontación entre lo propio y lo ajeno.

Gráfico 14. Actitud activa frente al texto

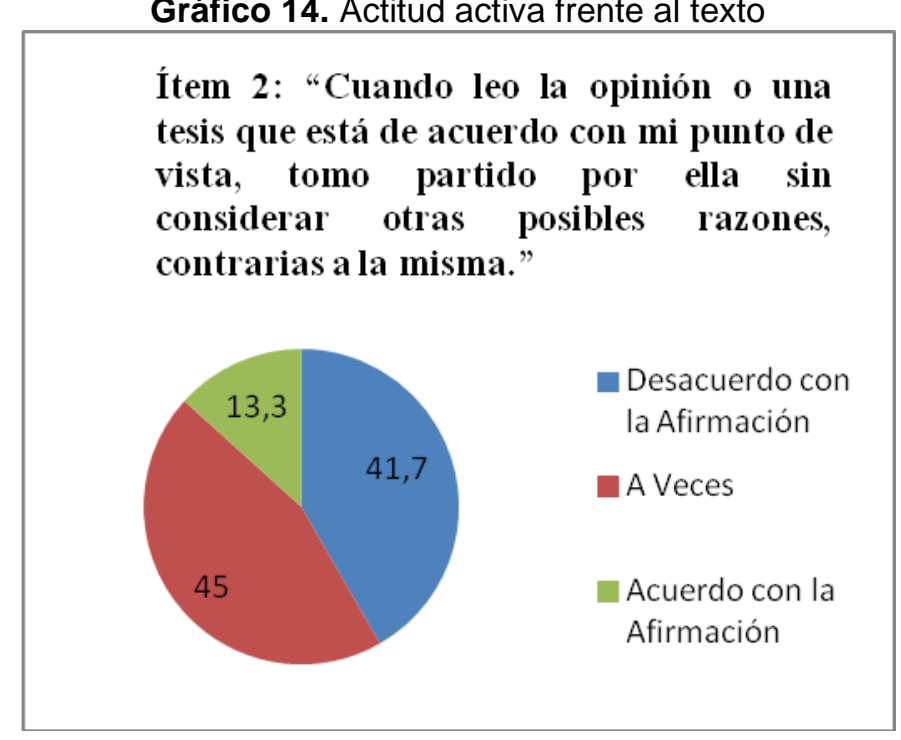

Fuente: Elaboración propia

Prácticamente el mismo porcentual de la muestra expresó no asumir posturas acríticas respecto de las expresiones vertidas en los textos o hacerlo en ocasiones. La búsqueda de 
argumentos que nutran una determinada posición es una cuestión sustantiva como habilidad lectora para la construcción del pensamiento crítico.

Gráfico 15. Actitud pasiva frente al texto

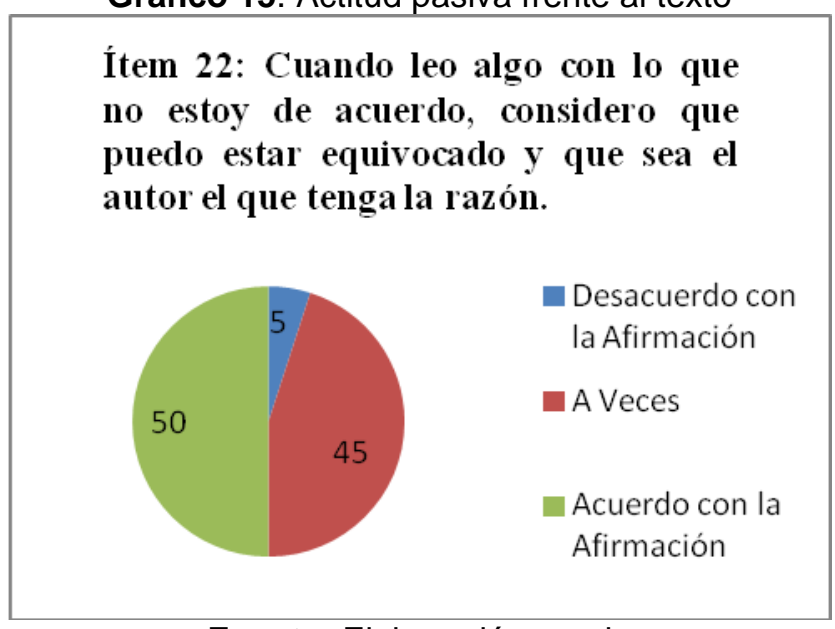

Fuente: Elaboración propia

La mitad de los encuestados priorizó la perspectiva del autor en desmedro de la propia y casi en igual proporción, lo hizo en ocasiones.

Gráfico 16. Búsqueda de argumentos

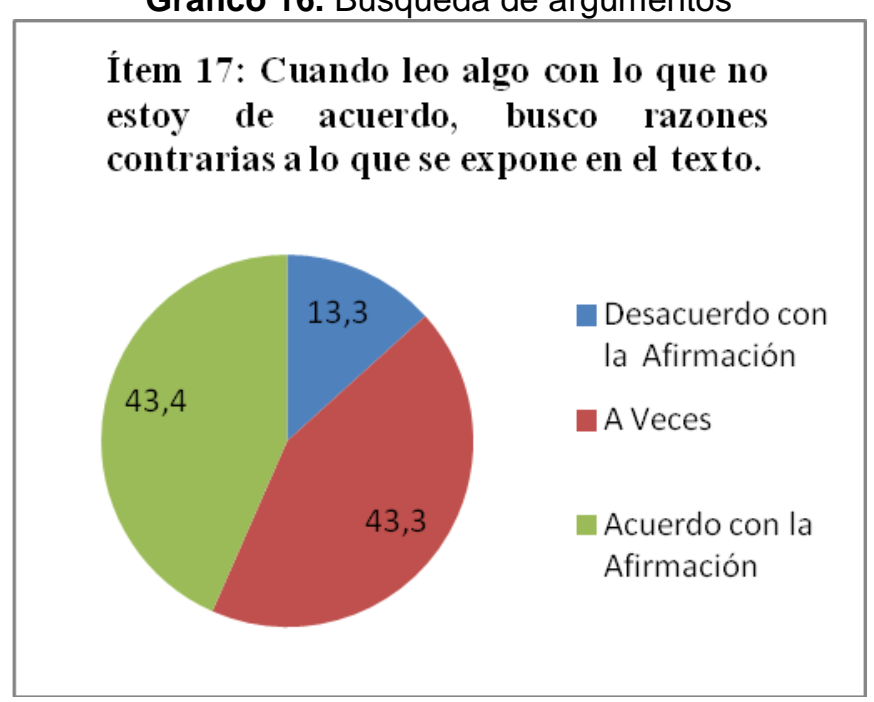

Fuente: Elaboración propia

Ante el desacuerdo con los textos, los encuestados afirmaron que de modo frecuente y en ocasiones, apelan a razones que invalidan la información expuesta en estos. 


\section{Discusión y conclusiones}

Los estudiantes de Ciencias de la Educación se autoperciben como lectores reflexivos en lo que hace a la identificación y diferenciación de las partes constituyente de un texto, tanto en los análisis previos, como en la lectura crítica en sí misma. No obstante, y en relación con este último aspecto, se perciben con mayor incertidumbre a la hora de confrontar entre tesis propias y ajenas.

Los encuestados significan positivamente aquellas habilidades vinculadas con la identificación y diferenciación de las partes de un texto académico.

El reconocimiento de las ideas principales y de los argumentos acerca de una tesis, son capacidades requeridas en esta etapa de la formación pues los géneros académicos o discursivos objeto de interpretación, son de tipo expositivo explicativo y argumentativo.

Los datos mostrarían una tendencia en la autopercepción, a favor de la capacidad de diferenciar conceptos sobre algo controvertido, aspectos por resolver y dar la razón o hacer patente los fundamentos de los aspectos planteados en textos académicos u otras fuentes. Cuestión no menor, ya que el alumno pone en juego estrategias lingüísticas y teóricas al distinguir meras opiniones de problemas o explicaciones científicas.

Estas capacidades de discriminación entre tipos de información, evidencias y status de enunciados (Curone et al, 2011) no ofrecen dificultades y permiten a los estudiantes la reconstrucción de su pensamiento (Paul y Elder, 2005).

En igual sentido, auto-perciben su capacidad para elaborar inferencias al examinar críticamente los textos, poniendo en juego procesos cognitivos que dan lugar a síntesis de los resultados más relevantes de un texto y a sentidos y significados diferentes a los expuestos por el/los autor/es, necesarios en la producción de textos académicos durante la formación y futura práctica profesional (Herrera-Ucrós y Villalba-Mercado, 2012).

Las habilidades de razonamiento deductivo-inductivo y analógico-abductivo contribuyen a la comprensión lectora (Lipman, 1998) y devienen en nuevas síntesis cognitivas. Las inferencias que los estudiantes atestiguan poner en acción son las de generalización e interpretación, al evaluar afirmaciones y atender la connotación de las palabras (Betancourth-Zambrano, Insuasti-Quevedo y Riascos-Portilla, 2012).

Valoran también contar con la habilidad para evaluar opciones como resultado de un análisis crítico conducente a la toma de decisiones con los consiguientes procesos cognitivos involucrados. Para ello, recurrirían a conocimientos e ideas externas al texto a efectos de relacionar dicha información con sus marcos de referencia conceptuales o metodológicos. 
En suma, en este nivel del trayecto de formación, los estudiantes al enfrentarse con lecturas propias del campo disciplinar y objetivar el texto despliegan estrategias de posicionamiento y estiman saber valorar las soluciones enunciadas, juicio crítico que requiere en cierta medida de la experiencia personal del lector. Son habilidades significativas como pensadores críticos (Ennis, 2002).

El poder distinguir algunos de los planos de la lectura crítica (Cassany, 2006) inferencias y presupuestos (entre líneas) entender la lógica interna textual y evaluar cuestiones que admiten discrepancias son habilidades de un buen lector en términos de Hawes, 2003).

Los encuestados otorgan relevancia tanto a la vigencia de teorías y conceptos como a la ponderación de evidencias para posicionarse respecto de tesis u opiniones, cuestiones sustantivas en la formación para una práctica profesional crítica y fundada, opinión que no aparece tan clara respecto de la capacidad de verificación de la lógica interna de los textos.

Esta tensión emerge también en relación con las percepciones en torno a sus habilidades para analizar componentes a efectos de acordar o no, como consecuencia de la actitud dubitativa en el rango de opiniones.

El señalamiento ocasional, respecto de la asunción crítica de la lectura, induce a preguntarse hasta dónde la evaluación de las tesis sostenidas por otros, impide que los estudiantes acepten sin cuestionar sus propuestas (Hawes, 2003).

Asimismo, la auto-percepción en relación con la confrontación de lo propio con lo ajeno, denota tensión entre el principio de autoridad y la propuesta de argumentos propios por parte de los estudiantes al disentir con el autor (principio de la experiencia) tal vez, por la actitud arraigada en una epistemología que equipara conocimiento con mera posesión de información. Esta habilidad requiere de la empatía intelectual, esto es, practicar pensar dentro del punto de vista de otros, especialmente de aquellos con los que no se concuerda (Paul y Elder, 2005).

La lectura en tanto proceso cognitivo complejo demanda destrezas intelectuales para el acceso a la información y construcción del conocimiento al poner en práctica las siguientes habilidades:

- Identificar y entender los contenidos explícitos de un texto.

- Comprender cómo se articulan las partes de un texto para darle un sentido global.

- Reflexionar a partir de un texto y evaluar su contenido.

- Reflexionar y valorar la forma de un texto. 
Por ende, la mirada crítica de un texto supone que el estudiante comprende las unidades básicas de sentido, integra dicha información para darle un sentido global y finalmente, asume una postura crítica acerca de su contenido.

Esta primera aproximación a las habilidades de lectura crítica percibidas por los estudiantes da cuenta de que los modos de operar con este tipo de lectura análisis previo, elaboración de inferencias y valoración sobre el texto, son consistentes con los hallazgos de Marciales-Vivas (2003) en estudiantes de carreras de índole multiparadigmática (Biglan, 1973).

Sin embargo, la ambigüedad en la valoración de las producciones propias y su confrontación con otros induce a pensar que estas habilidades de lectura crítica (Furedy y Furedy, 1985) concuerdan solo con algunos de los hallazgos de Curone (2005).

La mayoría de las habilidades de lectura crítica auto-percibidas por los estudiantes guardan vínculos con las características de carreras multiparadigmáticas (Ciencias de la Educación) que enfatizan la promoción de habilidades cognitivas de orden superior vinculadas con la identificación y evaluación de evidencias, el análisis de razones y fuentes, y la argumentación, cuestiones necesarias para el desarrollo de un pensamiento crítico. Esta última, no tan significativa en función de los valores mencionados.

Finalmente, dado que los resultados devienen de la autopercepción de los estudiantes valoración positiva, se estima pertinente su manejo con la reserva que el caso requiere, puesto que no realizaron tareas que den cuenta de análisis crítico, tópico a profundizar en futuras indagaciones con el fin de contar con evidencias de las habilidades de lectura crítica que afirman tener, y de las que realmente ponen en acción al operar con los textos académicos.

\section{Referencias}

Argudín, Yolanda y Luna, María. (1994). Aprendiendo a pensar leyendo bien. En habilidades de lectura a nivel superior (3ª ed.). México: Plaza y Valdés Editores.

Betancourth-Zambrano, Sonia, Insuasti-Quevedo, Katherine y Riascos-Portilla, Dadja. (2012). Pensamiento crítico a través de la discusión socrática en estudiantes universitarios. Revista Virtual Universidad Católica del Norte, (35). Recuperado de http://revistavirtual.ucn.edu.co/index.php/RevistaUCN/article/view/356

Biglan, Anthony. (1973). Relationships between subject matter characteristics and the structure and output of university departments. Journal of Applied Psychology, 57(3), 204-213. 
Candel Gutiérrez, Rosa Lidia y Pareja Candel, Mery Rocío. (2013). La lectura crítica en el proceso de enseñanza -aprendizaje del área de lengua y literatura de los estudiantes del quinto año de Educación General Básica de la escuela fiscal mixta "24 de mayo de la parroquia San Juan, cantón pueblo viejo, provincia de "Los Ríos" período lectivo 2012. (Tesis de Licenciadas en Ciencias de la Educación, mención Educación Básica), Universidad Estatal de Bolívar, Facultad de Ciencias de la Educación, Sociales, Filosóficas y Humanísticas, Escuela de Ciencias Básicas, Ecuador.

Cassany, Daniel. (2006). Tras las líneas. Sobre la lectura contemporánea. Barcelona: Anagrama.

Cunha, Gabriel, Mesquita, Isabel, Moreno, María Perla, Boleto, Antonio Fernando, Tavares, Tiago Manuel y Silva, Pedro Felipe (2010). Autopercepción de las competencias profesionales de los entrenadores de fútbol en función de la experiencia personal y de la formación académica. Cuadernos de Psicología del Deporte, 10(1), 23-36.

Curone, Gladys, Martinez-Frontera, Laura y Pesino, Carolina. (2005). Promoviendo Habilidades Cognitivas y Metacognitivas para el estudio universitario. Anuario de la XII Jornadas de Investigación. Primer Encuentro de Investigadores en Psicología del Mercosur. "Avances, nuevos desarrollos e integración regional". Facultad de Psicología, U.B.A. Buenos Aires.

Curone, Gladys, Alcover, Silvina, Pabago, Gustavo, Martinez-Frontera, Laura, Mayol, Juan de la Cruz y Colombo, María Elena. (2011). Habilidades de pensamiento crítico en alumnos ingresantes a la UBA que cursan la asignatura Psicología. Anuario de investigaciones, 18, 169-180.

Delgado Martínez, Amparo Carolina y Becerra Álvarez, Delcy Rocio. (2012). Descripción de la autopercepción en estudiantes de Psicología de la Universidad Pontificia Bolivariana Seccional Bucaramanga, sobre las competencias que desarrollan en sus procesos de formación. (Tesis para obtener el título de Psicología), Universidad Pontificia Bolivariana, Facultad de Ciencias Sociales, Escuela de Psicología. Bucaramanga, Colombia.

Ennis, Robert. (2002). An Outline of Goals for a Critical Thinking Curriculum and Its Assessment. Chicago, University of Illinois: 7. Recuperado de http://faculty.ed.uiuc.edu/rhennis.

Furedy, Christine y Furedy, John. (1985). Critical thinking. Toward research and dialogue. En Janet Gail Donald y Arthur Sullivan (eds.), Using Research to Improve Teaching and Learning 23, (pp. 51-69). Utah, USA: Willey Periodicals.

Galindo Ruiz de Chávez, María de los Ángeles. (2015). Lectura crítica hipertextual en la WEB 2.0. Revista Electrónica Actualidades Investigativas en Educación, 15(1), 1-29. DOI: dx.doi.org/10.15517/aie.v15i1.16972

Gómez Puerta, Lorena, Roca Uberes, Carles y Guerrero Solé, Frederic. (2014). ¿Cómo perciben los estudiantes la adquisición de competencias? Análisis comparado: Teorías de la Comunicación en la Universidad Pompeu Fabra. Historia y Comunicación Social. 19, 313-326. 
Herrera, Ucrós y Villalba, Mercado. (2012). Procesos de lectura crítica, mediación pedagógica para propiciar desarrollo de habilidades de pensamiento crítico en estudiantes universitarios. (Tesis de Maestría), Sistema de Universidades Estatales del Caribe Colombiano, Universidad de Sucre, Colombia.

Hymes, Dell. (1996). Acerca de la competencia comunicativa. Forma y función, 9, 13-37.

Hawes, Gustavo. (2003). Pensamiento crítico en la formación universitaria (Documento de Trabajo 2003/6 Proyecto Mecesup TAL 0101). Talca, Chile: Instituto de Investigación y Desarrollo Educacional de la Universidad de Talca.

Lipman, Matthew. (1998). Pensamiento complejo y educación. Madrid: Ediciones de la Torre.

Luke, Allan y Freebody, Peter. (1997). Shaping the Social Practices of Reading. En Sandy Muspratt, Allan Like and Peter Freebody (Eds.), Constructing Critical Literacies: teaching and learning textual practice (pp. 185-226). Sydney: Allen \& Unwin.

Maglio, Norma y Luque, Adriana. (2011). Definiéndome como estudiante: Cuestionario de autopercepción. 3er Congreso Internacional de Investigación. 15-17 de noviembre, La Plata. $\quad$ Recuperado http://www.memoria.fahce.unlp.edu.ar/trab eventos/ev.1373/ev.1373.pdf

Marciales-Vivas, Gloria Patricia. (2003). Pensamiento crítico: Diferencias en estudiantes universitarios en el tipo de creencias, estrategias e inferencias en la lectura crítica de textos. (Tesis Doctoral), Departamento de Psicología Evolutiva y de la Educación. Facultad de Educación. Universidad Complutense de Madrid, Madrid, España.

Martínez, Héctor. (2009). Autopercepción social y atribuciones cognoscitivas en estudiantes de bajo rendimiento académico. Electronic Journal of Research in Educational Psychology, 7(3), 1175-1216.

Masten, Ann y Coatsworth, Douglas. (1998). The development of competence in favorable and unfavorable environments. American Psychologist, 53(2) 205-220.

Mota de Cabrera, Carmen. (2010). Desarrollo del pensamiento crítico a través del discurso argumentativo: Una experiencia pedagógica en un curso de lectura y escritura. Entre Lengua, 15, 11-23.

Paul, Richard y Elder, Linda. (2003). La Mini-guía para el pensamiento crítico: conceptos y herramientas. Recuperado de https://www.criticalthinking.org/resources/PDF/SP_ConceptsandTools.pdf

Paul, Richard y Elder, Linda. (2005). Estándares de competencia para el pensamiento crítico. Estándares, Principios, Desempeño, Indicadores y Resultados. Con una Rúbrica Maestra en el Pensamiento Crítico. Recuperado de http://www.criticalthinking.org/resources/PDF/SP-Comp Standards.pdf 
Santiuste-Bermejo, Victor (coord.), Ayala-Flores, Carlos Luis, Barrigüete-Merchán, Carmen, García-García, Emilio, González-Pienda, Julio Antonio, Rossignoli, José Luis y Toledo, Enrique. (2001). El pensamiento crítico en la práctica educativa. Madrid: Fugaz Ediciones.

Santiuste Bermejo, Victor. (2001). Quelques réflexions sur la valur éducative de la Philosophie. Conferencia presentada en las Escuelas Europeas. Seminario de Filosofía. Bruselas, Bélgica.

Serrano, María Stella y Madrid, Alix. (2007). Competencias de lectura crítica. Una propuesta para la reflexión y la práctica. Acción Pedagógica, (16), 58-68.

Serrano de Moreno, Stella. (2008). El desarrollo de la comprensión crítica en los estudiantes universitarios. Hacia una propuesta didáctica. Revista Educere, 12(042), 505-514.

UNESCO. Conferencia Mundial Sobre La Educación. (1999). Declaración mundial sobre la educación superior en el siglo XXI: visión y acción y marco de acción prioritaria para el cambio y el desarrollo de la educación superior. Francia: UNESCO.

Vigotsky, Lev. (1985). Pensamiento y lenguaje. Buenos Aires: La Pléyade.

Yudkin, Ben. (2006). Critical Reading, Making sense of papers in life sciences and medicine. USA: New York. 